\title{
Patient-Reported Adverse Drug Reactions and Drug-Drug Interactions: A Cross-Sectional Study on Malaysian HIV/AIDS Patients
}

\author{
Syed S. Hasan ${ }^{a}$ See C. Keong ${ }^{a}$ Christopher L.K. Choong ${ }^{b}$ Syed I. Ahmed ${ }^{a}$ \\ Ting W. Ching ${ }^{\mathrm{b}}$ Mudassir Anwar $^{\mathrm{a}}$ Keivan Ahmadi $^{\mathrm{a}}$ Muneer G. Babar ${ }^{\mathrm{a}}$ \\ ${ }^{a}$ Department of Pharmacy Practice and Community Oral Health, School of Pharmacy and Dentistry, International

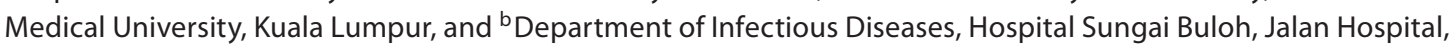 \\ Sungai Buloh, Malaysia
}

\section{Key Words}

Patient-reported adverse drug reactions $\cdot$ Drug-drug interactions · HIV/AIDS, Malaysians

\begin{abstract}
Objective: This study aimed to explore the adverse drug reactions (ADRs) reported by patients and to identify drugdrug interactions (DDIs) among human immunodeficiency virus/acquired immunodeficiency syndrome (HIV/AIDS) patients. Subjects and Methods: This cross-sectional study was conducted at the Medication Therapy Adherence Clinic, Hospital Sungai Buloh, an HIV/AIDS referral centre. The patients were randomly selected and were encouraged to describe ADRs caused specifically by any of the prescribed antiretroviral drugs (ARDs). Sociodemographic characteristics were recorded from the patients' medical records. In addition data on antiretroviral treatment (ART), DDIs and other conventional medication were also documented. Results: A total of 325 randomly selected HIV/AIDS patients with a mean age of 22.94 years participated in the study. The most frequently prescribed ARDs were lamivudine (64.6\%), zidovudine (40.6\%) and efavirenz (42.5\%). Commonly reported ADRs were fatigue (54.8\%), allergic reactions (41.5\%), weight loss (41.5\%), dry mouth (35.1\%) and memory loss (35.1\%). Female (87.8\%), non-complementary and alternative
\end{abstract}

medicine (CAM) users (87.3\%) and participants below 50 years old (81.1\%) were identified as having a higher prevalence of ADRs compared to males (79.6\%), CAM users (78.7\%) and participants aged 50 years or more (77.5\%). Patient age was found to be significantly associated $(p=0.048)$ with the ADRs. In addition, a total of 44 cases of DDIs belonging to category $\mathrm{D}$ were also found in this study. Conclusions: This study enabled us to identify the most common ADRs and DDIs associated with the use of ART. Safe and effective treatment depends on the healthcare providers' knowledge of the same.

Copyright $\odot 2011$ S. Karger AG, Basel

\section{Introduction}

Ever since the presence of human immunodeficiency virus (HIV) infection in central Africa in the year 1959, this incurable infection has changed the history of human health. According to the Joint United Nations Programme on HIV/Acquired Immunodeficiency Syndrome (AIDS), there were 33.2 million people living with HIV in the year 2007 with a daily increment of 6,800 HIV cases and about 5,700 cases of death from AIDS every day [1]. In Malaysia, a total of 60,621 HIV and 8,840 AIDS cases have been reported between 1986 and 2004 [2]. A 
survey in the USA reported that about $70 \%$ of individuals with HIV/AIDS were receiving antiretroviral treatment (ART) [3]. In Malaysia, the number of HIV/AIDS patients receiving ART has increased from 1,710 people in 2003 to 6,207 people in 2007 [4]. In 1996-1997 when ART was first introduced, approximately $50 \%$ of mortality reduction was shown among people living with HIV/AIDS who were compliant with the ART regimen [5].

In spite of the benefits associated with the use of ART, the issues of drug-induced toxicities such as fat redistribution, dyslipidaemia, sexual dysfunction, insulin resistance and diabetes have remained of great concern which may lead to the non-compliance and discontinuation of ART $[6,7]$. For instance the use of non-nucleoside reverse transcriptase inhibitors (NNRTIs) has been associated with rash and hepatotoxicity $[7,8]$. Nucleoside reverse transcriptase inhibitors (NRTIs) have been linked with hypersensitivity reactions, anaemia and neutropenia $[9,10]$. Protease inhibitors have also been associated with hyperlipidaemia, hyperglycaemia and gastrointestinal symptoms $[6,10,11]$. Furthermore, an increasingly complex drug therapy for HIV-infected patients not only increases the chances of adverse drug reactions (ADRs), but also provides ample potential for clinically significant drug interactions $[12,13]$. Besides drug-drug interactions (DDIs) between prescribed drugs, there is evidence that garlic, St. John's wort and vitamin C may reduce the concentration of antiretroviral drugs (ARDs) [14]. Numerous other studies have also reported an interaction of ARDs such as saquinavir and indinavir and complementary and alternative medicine (CAM) such as garlic and St. John's wort [15, 16]. Nevertheless, it has been reported that awareness of pharmacological interaction and toxicity is lacking in some regions such as Africa [14, 17]. However, this lack of awareness is not geographically confined to Africa, and it might be the same in other continents as well.

To our knowledge, there has been no published data regarding self-reported ADRs and the potential DDIs in Malaysian HIV/AIDS patients. Thus, the objectives of this research were formulated to explore the occurrence of ADRs and DDIs among HIV/AIDS patients receiving ART.

\section{Subjects and Methods}

This cross-sectional study was conducted on HIV/AIDS patients attending the Medication Therapy Adherence Clinic, Hospital Sungai Buloh, an HIV/AIDS referral centre, Malaysia. The calculated sample size was 377 with an estimated drop-out rate of $20 \%$ based on the total HIV/AIDS population $(95,000)$ in Malaysia, confidence level and confidence interval (CI). The simple ran- dom sampling technique was used, where randomization was carried out using patients' medical record numbers. Based on the randomization procedure adopted for this study, every fifth patient attending the clinic was selected from a pool of an average of 60 patients/day. A total of 400 HIV/AIDS patients attending the clinics was randomly approached. However, at the end of the data collection phase, 325 patients agreed to be interviewed. Study patients with tuberculosis were excluded so as to minimize the impact of confounding factors on the results. The sociodemographic factors of the patients attending the clinic were noted from the patients' medical records and were later confirmed with the individual patient at the start of the interview. In addition, data on ART, comorbidities and other conventional medication were also recorded. Only those patients who were on ART for at least 1 month were included in this study.

A 15- to 20-min interview was conducted at the Medication Therapy Adherence Clinic using the validated data collection form. The data collection form was distributed to 10 academic staff and 5 physicians to ascertain its content validity. The study information sheet was shown, and consent (either verbal or written) was taken from all the recruited patients prior to the interview. Subsequently, each participant was asked: 'Are you diagnosed as having HIV/AIDS?' and 'Are you here to see the doctor for HIV/AIDS?' It was done to ensure that the patient approached was the right patient to be interviewed. The patients attending the clinic were encouraged to describe ADRs caused specifically by any of the prescribed ARDs. In addition, confirmation of any action taken to resolve the adverse reactions, and treatment provided to manage the condition from the clinic were also recorded. They were also asked about the current use of any CAM.

The DDIs were also identified using 3 compendia: Micromedex, Hansten-Horn monographs and Lexi-comp online database. The classification provided in the Lexi-comp database is A, B, C, $\mathrm{D}, \mathrm{X}$; however, only $\mathrm{D}$ and $\mathrm{X}$ were considered clinically significant in this study. The recommendation for type $\mathrm{D}$ interactions was 'therapy modification' and for type X interaction 'contraindication'. Apart from ADRs and DDIs, risk factors associated with occurrence of ADRs were also identified.

Both descriptive (means and standard deviation) and inferential data analyses ( $\chi^{2}$ for categorical variables, Spearman correlation for non-normal distributed variables or Mann-Whitney for non-parametric data) were performed with the aid of SPSS ${ }^{\circledR}$ version 13.0, and variables were taken as statistically significant at $\mathrm{p} \leq 0.05$. Related odds ratios (ORs) and 95\% CIs allowed investigation of independent risk factors associated with ADRs. The study was approved by the research and ethics committee. In addition, permission was also granted from the director of the hospital, head of the infectious diseases department and pharmacy department. Information confidentiality was assured to all the study participants.

\section{Results}

\section{Sociodemographic Characteristics}

A total of 400 people living with HIV/AIDS were randomly approached at the Medication Therapy Adherence Clinic; however, only 325 patients agreed to be inter- 
Table 1. Demographic characteristics of the study population $(\mathrm{n}=325)$

\begin{tabular}{lcc}
\hline Variables & Number & Percent \\
\hline Gender & \multicolumn{2}{c}{} \\
Male & 284 & 87.4 \\
Female & 41 & 12.6 \\
Mean age, years & $22.94 \pm 9.114$ \\
Age groups & \multicolumn{2}{c}{} \\
18-30 years & 55 & 16.9 \\
31-49 years & 185 & 56.9 \\
$\geq 50$ years & 85 & 26.1 \\
Ethnicity & & \\
Malay & 87 & 26.8 \\
Chinese & 184 & 56.6 \\
Indian & 30 & 9.2 \\
Other & 24 & 7.4 \\
Marital status & & \\
Single & 188 & 57.8 \\
Married & 95 & 29.2 \\
Divorced & 29 & 8.9 \\
Widowed & 13 & 4.0 \\
Children & & \\
Yes & 105 & 62.3 \\
No & 220 & 67.7 \\
\hline
\end{tabular}

viewed. The mean age was found to be $22.94 \pm 9.1$ years. The age of more than half of the study participants (57\%) was between 31 and 49 years. The majority of the participants were male (88\%) and Chinese (56.6\%). Moreover, $58 \%$ were single and $68 \%$ had no children. The list of demographic characteristics is presented in table 1. Among the study participants, almost 231 (71\%) had received at least secondary school education. One hundred and sixtytwo $(50 \%)$ were labourers while $70(21.5 \%)$ were unemployed. The majority of the participants (43.4\%) disclosed their earnings between ringgit Malaysia (RM) 1,100 per month (USD 323) and RM 3,000 (USD 882). Two hundred and seventy (83\%) were staying in urban areas; 88 (27\%) were active smokers and 16 (4.9\%) were alcoholics.

\section{Utilization Pattern of ART}

All study participants were on ART, where lamivudine (64.6\%), zidovudine (40.6\%) and stavudine (23.7\%) were the top 3 prescribed NRTIs. Besides NRTIs, efavirenz $(42.5 \%)$ and nevirapine (19.4\%) were the only 2 NNRTIs prescribed to the participants; however, they were found to be more frequently used (61.8\%) than protease inhibitors (9.8\%). With ARD combination therapy, zidovudine, lamivudine in combination with efavirenz
Table 2. Record of patient adverse effect profile $(n=325)$

\begin{tabular}{lcc}
\hline Adverse effects & Number & Percent \\
\hline Fatigue & 178 & 54.8 \\
Allergy & 135 & 41.5 \\
Weight loss & 135 & 41.5 \\
Dry mouth & 114 & 35.1 \\
Memory loss & 114 & 35.1 \\
Giddiness & 98 & 30.2 \\
Insomnia & 91 & 28.0 \\
Headache & 89 & 27.4 \\
Heatiness & 82 & 25.2 \\
Nausea and vomiting & 71 & 21.8 \\
Loss of appetite & 65 & 20.0 \\
Diarrhoea & 45 & 13.8 \\
Nightmare & 43 & 13.2 \\
Stomach ache & 6 & 1.8 \\
Foot loss sensation & 6 & 1.8 \\
Cold sweat & 2 & 0.6 \\
Mood swing & 2 & 0.6 \\
\hline
\end{tabular}

(25.2\%) was the most frequent combination prescribed to the study participants. In addition, about $30 \%$ of the patients were using some form of CAM.

\section{Comorbidities and Other Conventional Drugs}

The most commonly found comorbidities in the study population were: hepatitis C, $16.6 \%$; dyslipidaemia, 9.5\%; toxoplasmosis, 8.3\%; hepatitis B, 8.0\%. Besides ART, the top 3 drugs most frequently prescribed were sulphamethoxazole-trimethoprim in $28.6 \%$, gemfibrozil in $6.5 \%$ and chlorpheniramine maleate in $4.6 \%$.

\section{Patient-Reported Adverse Effects and DDIs}

More than half of the study participants $(178,54.8 \%)$ reported to have fatigue after taking ART. The other frequently reported ADRs were allergic reactions (135, $41.5 \%)$, weight loss $(135,41.5 \%)$, dry mouth $(114,35.1 \%)$ and memory loss $(114,35.1 \%)$. The list of all ADRs reported by the study participants is presented in table 2 . Ten $(22.7 \%)$ cases of an interaction between gemfibrozil and pravastatin were identified. Apart from that, 7 (15.9\%) cases of an interaction between indinavir and ritonavir, and $4(9.1 \%)$ cases between itraconazole and efavirenz were also detected.

\section{Relationship of ADRs with Study Factors}

Gender (OR $=0.541, \mathrm{CI}=0.203-1.440)$, CAM use $(\mathrm{OR}=0.538, \mathrm{CI}=0.251-1.151), \mathrm{ART}(\mathrm{OR}=1.593, \mathrm{CI}=$ 
$0.896-2.834)$ and age less or more than 50 years $(\mathrm{OR}=$ $1.242, \mathrm{CI}=0.559-2.761$ ) were not found to be risk factors for the development of ADRs. However, age was found to be significantly associated $(\mathrm{p}=0.048)$ and correlated $(\mathrm{p}=$ $0.016)$ with the occurrence of ADRs.

\section{Discussion}

About $81 \%$ of the participants complained of experiencing ADRs, and this incidence rate was slightly higher compared to studies conducted by Sharma et al. [18] (71.1\%) and Johnson et al. [19] (70\%). The mean age of participants was 22.9 years which is significantly lower than in the study by Windi [20] in Sweden (44.8 years) and the study conducted by Peltzer et al. [17] in South Africa (34.0 years). The majority of the participants were aged between 31 and 49 years, and this is consistent with the statistics of the Ministry of Health, Malaysia, for the year 2008, where $42.2 \%$ HIV-positive people were aged between 30 and 39 years [21]. More than three quarters $(87.4 \%)$ of participants were male which reflects the overall picture of HIV/AIDS in Malaysia as $80.9 \%$ of HIV/ AIDS cases in 2008 were male [21]. Nevertheless, the numbers of females who were living with HIV/AIDS has noticeably increased about fivefold from 1986 to 2008 [21]. In this study, about $60 \%$ of the participants were Chinese; however, this picture did not reflect the real situation as Malays contributed to $67.5 \%$ of HIV and $56.8 \%$ of AIDS cases in the year 2006 [22].

In terms of socioeconomical characteristics, about $71 \%$ of the participants in this study were found to have at least a secondary education level compared with participants in Africa, in which only 29.9 and $20.5 \%$ of South Africans had at least a secondary school education [17, 23]. This is most likely due to the compulsory primary school education policy practice in Malaysia. Dyslipidaemia (9.5\%) was one of the comorbidities suffered by study participants. It is supported by a study carried out in Kenya, where $63.1 \%$ of the participants on stavudine, lamivudine and either nevirapine or efavirenz were diagnosed to have dyslipidaemia $[6,24]$. Similarly, several studies have reported that use of protease inhibitors could be associated with hyperlipidaemia $[6,18,19,25]$.

In Malaysia, any two drugs of choice from NRTIs in combination with any one drug of choice from NNRTIs are the first-line therapy. Common drugs of choice prescribed to study participants from the NRTI class were lamivudine (64.6\%), zidovudine (40.6\%) and stavudine (23.7\%), and this is almost similar to the report in 2003 (lamivudine, 46\%; zidovudine, 47\%; stavudine, 43\%) [26]. Currently, there are only 2 NNRTIs available in Malaysia, namely efavirenz and nevirapine. The current prescription rate of efavirenz is $42.5 \%$ compared to $68 \%$ reported by the National HIV/AIDS Treatment Registry. On the other hand, the prescription rate for nevirapine has increased from 6 to 19.4\% [26]. This might be due to the early introduction of efavirenz in the market (1999) compared to nevirapine (2001). Secondly it might also be due to the higher prevalence of rashes reported among people taking nevirapine than efavirenz $[7,10,11,24,26-$ 29]. In contrast to common ART practice in Taiwan, where $50.5 \%$ of the participants took an NRTI in combination with a protease inhibitor, most of the ART-receiving participants in this study were on NRTI in combination with NNRTI [18]. About 25\% of the ART-receiving participants (56.9\%) were taking zidovudine and lamivudine in combination with efavirenz. This is in line with the treatment guidelines by the Ministry of Health, Malaysia, which recommends the use of 2 NRTIs (zidovudine, lamivudine or stavudine) in combination with 1 NNRTI (efavirenz) [26]. A substantial number of patients (93) were on a sulphamethoxazole-trimethoprim combination for the treatment of pneumonia caused by Pneumocystis jiroveci (carinii), and this is particularly recommended when the CD4 count drops below 200 cells/ $\mu$ l.

The majority of ADRs described by the patients in this study could be disease originated; however, we emphasized on the report of ART-originated ADRs by patients. Similarly, ADRs such as hepatotoxicity and blood abnormalities may not have been reported by the patients in this study. Of all the reported adverse effects, fatigue (54.8\%) was the most common ADR reported by the study participants, and this is consistent with the studies done in South Africa (55.7\%) and Taiwan (65.1\%) [30, 31]. It has also been supported by Yeh et al. [30] where fatigue (54.9\%) was the most commonly experienced ADR by the participants taking an NNRTI in combination with an NRTI. Since zidovudine and lamivudine are considered as first-line therapy in Malaysia, it is not surprising that fatigue is the most commonly reported ADR. However, one should not exclude the possibility of psychologically induced fatigue, associated with HIV/AIDS. Allergic reactions constituted nearly $42 \%$ of the patients' reported adverse effects. Nonetheless, not all the reported cases were medically significant for the physicians as $16.3 \%$ of participants who reported allergic reactions were given anti-allergic medicines such as chlorpheniramine maleate. Skin rash was commonly reported among individuals taking stavudine (18-30\%), efavirenz (5-26\%) and ne- 
virapine (50\%) as the first-line therapy, which could explain the high occurrence of allergic reactions among the study participants [24]. Like previous studies, weight loss (41.5\%) was also a commonly reported ADR [32]. Poor appetite and mouth ulcers were the reasons reported by patients which could result in weight loss.

Higher incidences of ADRs were reported in participants who were on ART: $82.9 \%$ (189/228) of highly active ART users compared to $75.3 \%$ (73/97) of those not using highly active ART. Sharma et al. [18] stated that $50 \%$ of reported ADRs were related to zidovudine while stavudine contributed $47.9 \%$ of reported ADRs. This is in contrast with the study of Paterson et al. [32] where only $0.2 \%$ of participants complained of ADRs like cough and diarrhoea after ART initiation. In addition, females (87.8\%), non-CAM users $(87.3 \%)$ and participants below the age of 50 years $(81.1 \%)$ were identified as having a higher prevalence of ADRs compared to males (79.6\%), CAM users $(78.7 \%)$ and participants aged 50 years or above (77.5\%). One of the possible explanations for younger patients having a higher prevalence is their greater number in the study population.

\section{Conclusions}

The study could reflect a fair picture of the most commonly experienced ADRs with the use of ART in Malaysian HIV/AIDS patients. The uninformed and unpredictable ADRs may lead to non-adherence and ultimately treatment failure which poses a great necessity for the healthcare providers to be well equipped with the knowledge of ADRs and to convey this knowledge to the patients in order to optimize the adherence and eventually efficacy of the treatment. Co-administration of ART for the treatment of HIV/AIDS and drugs to treat other comorbid conditions increases the risk of DDIs as was evident in this study. Therefore, successful execution of a safe and effective therapy also relies on the healthcare providers' awareness on drug interaction potential, significance of interactions and strategy to cope when such interactions occur.

\section{References}

1 Joint United Nations Programme on HIV/ AIDS (UNAIDS). UNAIDS annual report. 2007. http://data.unaids.org/pub/Report/ 2008/jc1535_annual_report07_en.pdf (accessed August 14, 2009).

-2 Josephs JS, Fleishman JA, Gaist P, Gebo KA: Use of complementary and alternative medicines (CAM) among a multistate, multisite cohort of people living with HIV/AIDS. HIV Med 2007;8:300-305.

-3 Mokhtar N, Chan SC: Use of complementary medicine amongst asthmatic patients in primary care. Med J Malaysia 2006;61:125-127.

4 Remli R, Chan SC: Use of complementary medicine in systemic lupus erythematosus patients in Malaysia. APLAR J Rheumatol 2003;6:21-25.

5 Palella FJ, Delaney KM, Moorman AC, Loveless MO: Declining morbidity and mortality among patients with advanced human immunodeficiency virus infection. $\mathrm{N}$ Engl J Med 1998;338:853-860.

6 Nachega JB, Trotta MP, Nelson M, Ammassari A: Impact of metabolic complications on antiretroviral treatment adherence: clinical and public health implications. Curr HIV/ AIDS Rep 2009;6:121-129.

7 Minzi OMS, Irunde H, Moshiro C: HIV patients presenting common adverse drug events caused by highly active antiretroviral therapy in Tanzania. Tanzan J Health Res 2009;11:5-10.
-8 Knobel H, Guelar A, Montero M, Carmona A, Luque S, Gonzalez A: Risk of side effects associated with the use of nevirapine in treatment-naïve patients, with respect to gender and CD4 cell count. HIV Med 2008; 9:14-18.

-9 Taha TE, Kumwenda N, Kafulafula G, Kumwenda J, Chitale R, Nkhoma C: Haematological changes in African children who received short-term prophylaxis with nevirapine and zidovudine at birth. Ann Trop Paediatr 2004;34:301-309.

10 Vigouroux C, Gharakhanian S, Salhi Y, Nguyên TH, Adda N, Rozenbaum W: Adverse metabolic disorders during highly active antiretroviral treatments (HAART) of HIV disease. Diabetes Metab 1999;25:383392.

11 Nuesch R, Srasuebkul P, Ananworanich J, HIV-NAT Study Team: Monitoring the toxicity of antiretroviral therapy in resource limited settings: a prospective clinical trial cohort in Thailand. J Antimicrob Chemother 2006;58:637-644.

12 Miller CD, El-Kholi R, Faragon JJ, Lodise TP: Prevalence and risk factors for clinically significant drug interactions with antiretroviral therapy. Pharmacotherapy 2007;27: 1319-1386.
13 Robertson SM, Penzak SR, Pau A: Drug interactions in the management of HIV infection: an update. Expert Opin Pharmacother 2007;8:2947-2963.

14 Mills E, Ping W, Ernst E: Complementary therapies for the treatment of HIV: in search of the evidence. Int J STD AIDS 2005;16: 395-402.

15 Piscitelli BC, Burstein AH, Welden N, Gallicano K, Fallon J: Garlic supplement decreases saquinavir plasma concentrations (abstract). Proc 8th Conf Retroviruses Opportunistic Infect, Chicago, February 2001.

16 Piscitelli SC, Burstein AH, Chaitt D, Alfaro RM, Fallon J: Indinavir concentrations and St John's wort. Lancet 2000;355:547-548.

17 Peltzer K, Preez NF, Ramlagan S, Fomundam H: Use of traditional complementary and alternative medicine for HIV patients in KwaZulu-Natal, South Africa. BMC Public Health 2008;8:255

18 Sharma A, Vora R, Modi M, Sharma A, Marfatia Y: Adverse effects of antiretroviral treatment. Indian J Dermatol Venereol Leprol 2008;74:234-237.

19 Johnson MO, Stallworth T, Neilands TB: The drugs or the disease? Causal attributions of symptoms held by HIV-positive adults on HAART. AIDS Behav 2003;7:109-117.

20 Windi A: Determinants of complementary alternative medicine (CAM) use. Complement Ther Med 2004;12:99-111. 
21 Malaysian AIDS Council: 2008 Annual Report. 2008. http://www.mac.org.my/attachment/report2008.pdf (accessed August 15, 2009).

22 Ministry of Health Malaysia, Department of Public Health, Section of AIDS/STD: Summary of HIV/AIDS cases reported by the year 2006. Putrajaya, Ministry of Health Malaysia, 2007.

23 Klassen DL, Kipp W, Jhangri GS, Rubaale T: Use of traditional herbal medicine by AIDS patients in Kabarole District, Western Uganda. Am J Trop Med Hyg 2007;77:757-763.

24 Manuthu EM, Joshi MD, Lule GN, Karari E: Prevalence of dyslipidemia and dysglycaemia in HIV infected patients. East Afr Med J 2008;85:7-10.
25 Makoae LN, Seboni NM, Molosiwa K, Moleko M, Human S, Sukati NA: The symptom experience of people living with HIV/ AIDS in Southern Africa. J Assoc Nurses AIDS Care 2005;16:22-32.

26 Christopher Lee KC: The first report of the National HIV/AIDS Treatment Registry 2003. Kuala Lumpur, National HIV/AIDS Treatment Registry, 2005.

27 Bica L, McGovern B, Dhar R, Stone D, McGowan K, Scheib R: Increasing mortality due to end-stage liver disease in patients with human immunodeficiency virus infection. Clin Infect Dis 2001;32:492-497.

28 Monga HK, Rodriguez-Barradas MC, Breaux K, Khattak K, Troisi CL, Velez M: Hepatitis $\mathrm{C}$ virus infection-related morbidity and mortality among patients with human immunodeficiency virus infection. Clin Infect Dis 2001;33:240-247.
9 Simone MJ, Appelbaum J: HIV in older adults. Geriatrics 2008;63:6-12.

30 Yeh YL, Liu HY, Ko WC, Lee HC, Ko NY: HIV related symptoms in patients with HIV infection enrolled in an HIV case management program in Taiwan. J Nursing 2008; 16 : 237-245.

1 Standish LJ, Greene KB, Bain S, Reeves C, Sanders F, Wines RCM: Alternative medicine use in HIV-positive men and women: demographics, utilization patterns and health status. AIDS Care 2001;13:197-208.

32 Paterson DL, Swindells S, Mohr J, Brester M, Vergis EN, Squier C: Adherence to protease inhibitor therapy and outcomes in patients with HIV infection. Ann Intern Med 2000; 133:21-30. 